\title{
Linfoma primario del sistema nervioso central de la fosa posterior en un paciente joven
}

\author{
Tatiana RoldáN ${ }^{1}$, ÓsCar ZORRO $^{2}$, LuIs María Villalobos $^{3}$, Lina María RodríGueZ ${ }^{4}$
}

\section{Resumen}

El linfoma primario del sistema nervioso central es una neoplasia rara que se encuentra con relativa frecuencia en pacientes con inmunosupresión.

Se presenta el caso de una mujer de 38 años sin antecedentes relevantes, a quien se le resecó un tumor del ángulo ponto-cerebeloso izquierdo, cuya anatomopatología reportó un linfoma B de célula grande muy maligno. Se hicieron estudios en busca de inmunosupresión, los que fueron negativos. La paciente no se encontraba en el rango de edad de presentación de este tipo de tumor y sólo estudios moleculares genéticos podrían dar alguna explicación a este caso.

Palabras clave: linfoma primario, ángulo pontocerebeloso, sistema nervioso central.

\section{Title}

Primary central nervous system lymphoma of posterior fossa in young patient

\begin{abstract}
Primary central nervous system lymphoma is a rare condition which commonly presents in immunosuppressed patients.
\end{abstract}

1 Residente Medicina de Urgencias, Facultad de Medicina, Pontificia Universidad Javeriana. HUSI, Bogotá, D.C., Colombia.

2 Neurocirujano, Hospital universitario San Ignacio, Bogotá, D.C., Colombia.

3 Residente Neurocirugía, Facultad de Medicina, Pontificia Universidad Javeriana, Bogotá, D.C., Colombia.

4 Interna XI semestre, Facultad de Medicina, Pontificia Universidad Javeriana, Bogotá, D.C., Colombia. 
We present the case of a 38 year old female with no relevant previous medical history who underwent surgery for the removal of a mass located in her left cerebello pontine angle. Final pathology report was consistent with B - cell lymphoma (large cell variant). A survey for hidden conditions which could predispose the patient to immunodeficiency was negative. The age of presentation for this case was also atypical. Further genetic and molecular testing could provide a possible explanation for this rare event.

Key words: primary lymphoma, cerebellopontine angle, central nervous system.

\section{Introducción}

El linfoma primario del sistema nervioso central es un linfoma no Hodgkin que por muchos años fue conocido como microglioma, sarcoma de células reticulares o sarcoma perivascular, cuyo origen de células malignas linfocíticas ahora está bien establecido.

$\mathrm{Su}$ incidencia ha incrementado notoriamente en los últimos 10 a 15 años, debido al aumento de la infección por el virus de la inmunodeficiencia humana (VIH) y de la tasa de supervivencia de los pacientes con sida[1]. Se presenta en todos los grupos de edad, aunque la mayor incidencia está entre los 60 y los 70 años en individuos inmunocomprometidos, con una edad media de diagnóstico a los 55 años.

La presentación clínica depende de la localización del tumor, pero muchos pacientes $(50 \%)$ presentan síntomas relacionados con el efecto de masa intracraneal, principalmente déficit motor y sensitivo focales. Debido a que este tumor frecuentemente com- promete el lóbulo frontal, es común encontrar cambios en la personalidad. Raramente, se presentan síndrome extrapiramidal o signos y síntomas de tallo cerebral o cerebelo como primera manifestación de la enfermedad[2].

En el linfoma primario del sistema nervioso central se observa una morfología similar a la del linfoma no Hodgkin, pero difiere de esta entidad en su comportamiento biológico y molecular. Aproximadamente, 90\% son linfomas B de célula grande de alto grado de malignidad y el $10 \%$ son linfomas pobremente diferenciados de bajo grado, linfoma de Burkitt y linfomas de células T[3].

Los hallazgos en los estudios radiológicos (tomografía computadorizada y resonancia magnética) no son patognomónicos, pero el diagnóstico puede sospecharse con base en la apariencia de la lesión. En la tomografía computadorizada sin contraste, el tumor generalmente es isodenso o hiperdenso, mientras que, en la secuencia $\mathrm{T} 2$ de la resonancia magnética, es isointenso o hipointenso.

\section{Reporte de caso}

Se presenta el caso de una mujer de 38 años que consultó al Servicio de Urgencias del Hospital San Ignacio, remitida de otra institución, con cuadro clínico de dos meses de evolución, consistente en acúfenos (tinnitus) izquierdo y, posteriormente, parálisis fa- 
cial periférica izquierda, vértigo, pérdida de la agudeza auditiva izquierda y marcha inestable, sin cefalea. La paciente no tenía antecedentes personales o familiares relevantes.

En el examen físico de ingreso se encontró una paciente con signos vitales normales, hidratada y sin hallazgos positivos a la auscultación cardiopulmonar. En el examen neurológico, se obtuvo un examen mental normal, y se encontraron pupilas simétricas, normorreactivas, parálisis facial periférica izquierda de grado $\mathrm{V}$ en la escala de House-Brackmann, hipoacusia neurosensorial izquierda, reflejo nauseoso izquierdo disminuido, fuerza y reflejos músculo-tendinosos sin alteraciones, signo de Babinski positivo del lado izquierdo, dismetría y disdiadococinesia izquierdas y ataxia troncular. Con estos hallazgos se hizo el diagnóstico de un síndrome cerebeloso hemisférico izquierdo y síndrome de alteración de pares craneales VII, VIII y IX izquierdos.

Se obtuvieron imágenes de resonancia magnética cerebral simple y con gadolinio, que confirmaron la localización de la lesión en el pedúnculo cerebeloso medio izquierdo, que se extendía a la cara lateral del puente y la cara tentorial del hemisferio cerebeloso del mismo lado, con realce homogéneo con el medio de contraste, sin efecto compresivo importante, el cuarto ventrículo permeable y sin hidrocefalia (figura 1).

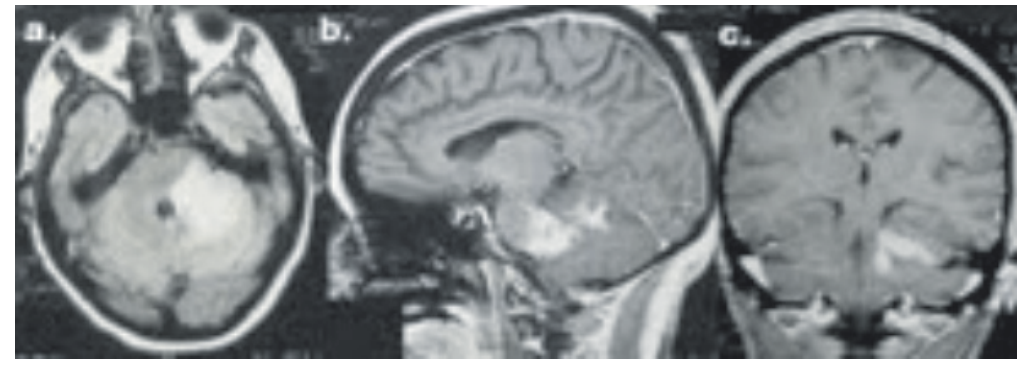

Figura 1. A. Imagen de resonancia magnética cerebral, secuencia FLAIR, cortes axiales, que muestran lesión con importante edema vasogénico y citotóxico, localizada en el pedúnculo cerebeloso medio izquierdo, y se extendía a cerebelo y puente. B y C. Secuencias en T1 con gadolinio, cortes sagitales y coronales, que muestran realce homogéneo con el medio de contraste de la lesión descrita. 
La paciente fue hospitalizada para manejo quirúrgico y se sometió a resección del tumor de la fosa posterior, por craneotomía suboccipital retromastoidea izquierda (figura 2). Los hallazgos intraoperatorios mostraron una lesión ricamente irrigada, rosadogrisácea, que podía aspirarse fácilmente en algunas porciones y con otras porciones más sólidas.
El espécimen quirúrgico fue llevado al Servicio de Patología, en donde se hizo estudio con tinciones convencionales que mostró un parénquima cerebeloso de aspecto histológico aparentemente usual, sin lesiones tumorales ni inflamatorias. La paciente presentó una evolución posquirúrgica satisfactoria con el manejo intravenoso de corticoides, por lo cual fue dada de alta.

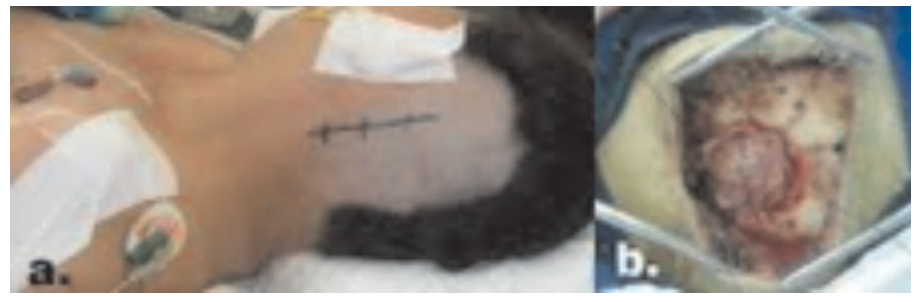

Figura 2. A. Posición de la paciente en decúbito supino con rotación de la cabeza a la derecha, rasurado y marcación de la herida según el planeamiento quirúrgico.

B. Craneotomía suboccipital retromastoidea izquierda con apertura de la duramadre, con exposición del cerebelo tenso por el edema asociado a la lesión.

Regresó a los 20 días al Servicio de Urgencias, por sintomatología respiratoria aguda y se le hizo diagnóstico de tromboembolismo pulmonar que fue manejado por el Servicio de Medicina Interna. Evolucionó con cefalea persistente tras el destete de la corticoterapia parenteral, por lo que fue valorada nuevamente por neurocirugía.
Se hizo estudio de imágenes de control que mostraron crecimiento de la lesión, por lo cual fue llevada nuevamente a cirugía con un grado de resección más agresivo. Se hicieron tinciones adicionales que permitieron el diagnóstico de linfoma $B$ de célula grande de alto grado de malignidad (figura 3). 


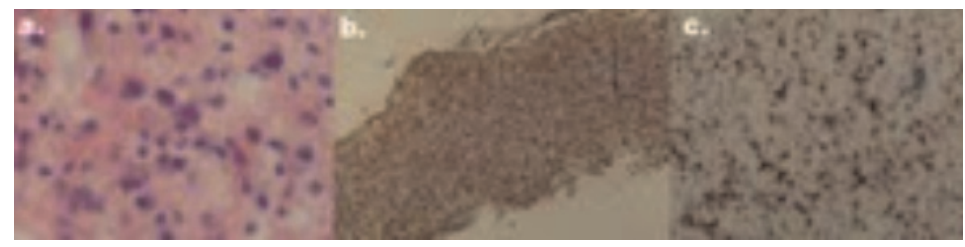

Figura 3. A. Se observan grupos de células grandes de núcleos ovales y abundante citoplasma eosinófilico. Hematoxilina eosina. B. Se aprecia reacción del 100\% de las células tumorales para el marcador de inmunoperoxidasa antígeno común leucocitario.

C. Se observa reacción de las células tumorales para el marcador de inmunoperoxidasa Ki- 67 (índice de proliferación) en $75 \%$ de las células tumorales. (Servicio de Patología, Hospital

Universitario San Ignacio).

La paciente continuó con el manejo complementario de radioterapia y quimioterapia, pero presentó una complicación infecciosa por neumonía hospitalaria, producto de la disfunción de pares craneanos bajos; empeoró y presentó neutropenia febril y choque séptico, y finalmente, falleció.

\section{Discusión}

El linfoma primario del sistema nervioso central es una neoplasia poco frecuente que constituye sólo $2 \%$ a $3 \%$ de los casos de linfoma no Hodgkin y $4 \%$ de los tumores cerebrales primarios. Más del $90 \%$ son de tipo linfoma $\mathrm{B}$ de célula grande. Tiene mayor prevalencia en el sexo masculino, en mayores de 60 años, y no se conocen diferencias raciales en la incidencia[4].

Múltiples estudios sugieren que la incidencia se encuentra actualmente en aumento y este cambio no está rela- cionado con el incremento de los trasplantes de órganos o los avances tecnológicos en el diagnóstico, sino con la elevada incidencia de sida[5]. Los pacientes inmunocomprometidos presentan mayor riesgo de desarrollar este tipo de neoplasia, la cual se observa con mayor frecuencia con la presencia de VIH, con trasplante de órganos y con síndromes de inmunodeficiencia congénita.

En este contexto, el linfoma primario del sistema nervioso central puede tener como origen la infección por el virus de Epstein-Barr que afecta los linfocitos $\mathrm{B}$, los cuales proliferan de manera desenfrenada con tendencia a formar tumores[6]. Existe especial interés en los reportes de nuevos virus herpes, como el virus humano herpes 8 , o el virus herpes humano asociado al sarcoma de Kaposi, en pacientes inmunocomprometidos. Estos virus juegan un papel importante en la gé- 
nesis del sarcoma de Kaposi y de linfomas que comprometen pleura, pericardio y peritoneo (linfomas de cavidades corporales)[7].

El virus de Epstein-Barr y el virus del herpes han sido investigados, pero aún no se ha encontrado una correlación exacta con el desarrollo de neoplasias en el sistema nervioso central, por lo cual continúa siendo un misterio. Tampoco se ha establecido una causa para el linfoma primario del sistema nervioso central en pacientes inmunocompetentes[8], ni se ha encontrado asociación viral con la patogénesis. En este grupo, el desarrollo de dichos tumores parece tener un origen diferente, que estaría relacionado con factores genéticos como la expresión alterada del gen $p 53$, según estudios moleculares[9].

La biopsia es indispensable para hacer el diagnóstico con exactitud y facilitar la selección del tratamiento definitivo. La sensibilidad diagnóstica puede llegar hasta 80\%[5]. El linfoma primario del sistema nervioso central de bajo grado es raro, está asociado con síntomas leves o atípicos, y está localizado en la médula espinal[4].

En cuanto a las manifestaciones clínicas, típicamente, los pacientes presentan síntomas sugestivos de disfunción neurológica focal que dependen de la localización del tumor. Predominantemente, se desarrollan cambios cognitivos, de comportamiento y personalidad, que están re- lacionados con la frecuente localización de este linfoma en el cuerpo calloso y en los lóbulos frontales.

En la literatura se han identificado factores pronósticos para linfomas no Hodgkin, que incluyen los niveles séricos de ácido láctico, la edad, la escala de Karnofsky, la elevación de proteínas en el líquido cefalorraquídeo y la localización del tumor en estructuras profundas.

El manejo clínico de esta enfermedad permanece controvertido y su tratamiento óptimo aún no se ha definido. El conocimiento actual sobre estrategias terapéuticas para el linfoma primario del sistema nervioso central resulta de un número pequeño de estudios prospectivos, metanálisis y series retrospectivas multicéntricas. El metotrexato en dosis altas se considera un medicamento efectivo, debido a su excelente actividad y penetración en el sistema nervioso central; puede usarse solo o en combinación con otros medicamentos o radioterapia[11]. La tasa de respuesta a la radioterapia es de $90 \%$, aproximadamente, pero se ha reportado una tasa inaceptablemente elevada de neurotoxicidad después del tratamiento (83\% a un año en pacientes mayores de 60 años)[12], por lo cual se ha incrementado el interés por altas dosis de quimioterapia que no requieren irradiación adicional.

Por otra parte, el tratamiento quirúrgico con resección total o parcial no ha demostrado mejorar la supervi- 
vencia debido a la naturaleza multifactorial e infiltrante del linfoma primario del sistema nervioso central y, además, la localización profunda de la mayoría de estos tumores puede generar secuelas neurológicas graves e irreversibles[3].

\section{Conclusión}

El linfoma primario de sistema nervioso central es una forma inusual de linfoma no Hodgkin y aún más inusual es su presentación en el cerebelo. Es un tumor que, aunque se presenta con síntomas neurológicos focales, histológicamente está caracterizado por infiltración difusa del parénquima cerebral; su manejo óptimo aún no se ha establecido pero el tratamiento combinado parece mejorar la supervivencia de los pacientes.

Este caso que se presenta es interesante, pues la paciente estaba fuera del rango de edad usual para linfoma primario del sistema nervioso central y no se encontraba inmunosuprimida, y la localización en la fosa posterior es muy inusual. Las alteraciones moleculares en la expresión de oncogenes pudieran dar luz a muchas de las dudas despertadas con este caso.

\section{Bibliografía}

1. Engels E, Biggar R, Hall H, Cross H, Crutchfield A, Finch J, Grigg R, Hylton T, Pawlish K, McNeel T, Goedert J. Cancer risk in people infected with human immunodeficiency virus in the United. Int J Cancer. 2008;123:187-94.

2. Chimienti E, Spina M, Vaccher E, Tirelli $\mathrm{U}$. Management of immunocompetent patients with primary central nervous system lymphoma. Clin Lymphoma Myeloma. 2009;5:353-64.

3. Batchelor T, Loeffler JS. Primary CNS lymphoma. J Clin Oncol. 2006;24:1281-8.

4. Rubenstein J, Ferreri A, Pitaluga S. Primary lymphoma of the central nervous system: Epidemiology, pathology and current approaches to diagnosis, prognosis and treatment. Leuk Lymphoma. 2008;49(Suppl.1):43-51.

5. Olson JE, Janney CA, Rao RD, Cerhan JR, Kurtin PJ, Schiff D, et al. The continuing increase in the incidence of primary central nervous system nonHodgkin lymphoma: A surveillance, epidemiology, and end results analysis. Cancer. 2002;95:1504-10.

6. Mohile N, Abrey L. Primary central nervous system lymphoma. Neurol Clin. 2007;25:1193-207.

7. van der Valk P. Central nervous system lymphomas. Curr Diagn Pathol. 1996; 3:45-52.

8. Jahnke K, Hummel M, Korfel A, Burmeister T, Kiewe P, Klasen HA, Müller HH, Stein H, Thiel E.. Detection of subclinical systemic disease in primary CNS lymphoma by polymerase chain reaction of the rearranged immunoglobulin heavy chain genes. $J$ Clin Oncol. 2006;24:4754-7.

9. Koga H, Zhang S, Ichikawa $\mathrm{T}$, Washiyama K, Kuroiwa T, Tanaka R, Kumanishi T. Primary malignant 
lymphoma of the brain: Demonstration of the $p 53$ gene mutations by PCRSSCP analysis and immunohistochemistry. Brain Tumor Pathol. 1994;11:151-5.

10. Ferreri AJ, Reni M, Pasini F, Calderoni A, Tirelli U, Pivnik A, et al. A multicenter study of treatment of primary CNS lymphoma. Neurology. 2002;58:1513-20.

11. Ferreri AJ, Abrey LE, Blay JY, Borisch B, Hochman J, Neuwelt EA, et al.
Summary statement on primary central nervous system lymphomas from the Eighth International Conference on Malignant Lymphoma, Lugano, Switzerland, June 12 to 15,2002 . J Clin Oncol. 2003;21:2407-14.

12. Abrey L, Yahalom J, De Angelis L. Treatment for primary CNS lymphoma: The next step. J Clin Oncol. 2000; 18:3144-50. 